\title{
Healthy lifestyle habits benefit remission of recurrent aphthous stomatitis and RAS type ulceration
}

\author{
L. Ge
}

In brief

Highlights that good habits benefit health, bad

habits lead to diseases.

Suggests mental health might have a decisive effect

on recurrent aphthous stomatitis (RAS).

Suggests lifestyle habits, which promote mental health, benefit remission of RAS and RAS type ulceration.

In a clinical setting, it is not practical to separate recurrent aphthous stomatitis (RAS) from RAS-type ulceration in every case, so both conditions have been considered together for the purpose of this article. Due to the lack of consistently effective therapeutic options for RAS, other approaches to treat the disease are considered here, beginning with an analysis of possible aetiological factors. The aetiology of RAS, the most common mucosal disease, remains undefined thus far. A few researchers have proposed psychological disorders as a major factor impacting RAS. Some systemic diseases and lifestyle habits are also reported to be associated with RAS; these also impact mental health. I believe that all habits contributing to mental health might also benefit patients with RAS. I suggest that practitioners of oral medicine should recommend a healthy lifestyle to patients with RAS, before prescribing medicines.

Oral mucosal diseases like recurrent aphthous stomatitis (RAS) are a common presenting condition in certain hospitals served as the centres for treating oral mucosal diseases in China, and can tie-up substantial healthcare resources. It is not always practical to distinguish between RAS and RAS-type ulceration in every case in a clinical setting; therefore, a series of investigations must be prescribed to exclude systemic diseases associated with RAS. Patients expect faster relief for such conditions and may not always be agreeable to such investigations which can involve long waiting periods and incur a high cost to the patient, for which they might not get reimbursed. Therefore, unless the symptoms of associated systemic diseases are obvious, physicians do not distinguish between RAS and RAS-type ulceration. For this reason, both conditions have been considered together here. A Cochrane review on the efficacy of therapies for RAS did not report any consistently effective therapy. ${ }^{1}$ Given the large number

State Key Laboratory of Oral Diseases, National Clinical Research Center for Oral Diseases, Department of Oral Medicine, West China School of Stomatology, Sichuan University Department of Oral Medicine, West China Hospital of Stomatology, Sichuan University

Correspondence to: Dr Lin Ge.

Email:v1i2c3@163.com

Refereed Paper.

Accepted 18 September 2017

Published online 12 January 2018

DOI: $10.1038 /$ sj.bdj.2018.38 of patients discomfited by RAS, the question of finding an effective treatment to provide relief to patients suffering from this chronic, recurrent disorder is a valid one.

Using lifestyle as a keyword, over 100 articles were retrieved by a search in the archives of New England Journal of Medicine. The most recent article was published on July 132017 and the earliest on September 16 1971. There were 20-30 articles published in every ten year period, including reviews. In the period 1971 to 1974 , the articles did not report specific therapeutic research and mainly discussed the lifestyle of different affected groups such as (female) physicians, elderly patients of tuberculosis, and drug addicts. The only research article published in 2017 used a database containing a large patient sample with long-term follow-up to observe the relationship between the quality of diet and mortality. ${ }^{2}$ In addition to general information on death, health etc, the impact of lifestyle on specific diseases such as cardiovascular disorders, diabetes, psychological disorders, cancer, and other diseases was also discussed. A typical disease would be gestational diabetes mellitus (GDM); in many cases, changes in lifestyle (such as dietary control and more exercise) were reported to bring GDM under control. Thousands of years ago, the Inner Canon of the Yellow Emperor, the most authoritative work on Chinese traditional medicine (CTM), reflected on the idea of preventive healthcare:
'The ancient people, who knew Tao, could follow the law of Yin and Yang and coordinate Shushu; they were moderate in their consumption of food and drink, had regular sleep and wake patterns, and did not overwork; these habits helped them maintain their mental and physical well-being, helping them to live long lives, even over 100 years.'

Correct lifestyle habits could prolong one's life. The work also states:

'The onset of numerous diseases is caused by wind and rain (signifying adverse climate), cold and heat (signifying extremes of temperature), cold and humidity (two of the six external factors for disease mentioned in the text), and joy and anger (signifying internal factors/mood)'.

[Note: There is controversy about the book age of Inner Canon of the Yellow Emperor. The earliest controversial time is in the pre-Qin period (21st century BC $-221 \mathrm{BC}$ ), the latest in the Western Han Dynasty (202 BC - AD 8).]

A proper balance of internal and external factors can help prevent many diseases. Inappropriate lifestyle habits could have an impact on the occurrence of diseases. In the same way, lifestyle changes rather than medicine could probably contribute to long-term remission in RAS.

Some researchers have reported a relationship between intestinal disorders and RAS-type ulceration. Gluten protein is one of the most complex protein systems, consisting of components of many different sizes. Moreover, 
genotypes, growth conditions, and technological progress also affect its complexity. Gluten has been implicated in the pathology of many clinical conditions, including gluten allergy and parenteral disorders known as non-coeliac gluten sensitisation (NCGS). Ferguson et al. investigated jejunal mucosal abnormalities via biopsies in patients with RAS and found that samples from most patients (30/33) had a flat mucosa. They reported that gluten sensitivity might cause histological changes to the jejunal mucosa and RAS could remit with a gluten-free diet (GFD). ${ }^{3}$ The authors suggested that milk protein, etc. could also increase the risk of RAS. Di Sabatino et al. used self-controlled trials to observe the effects of low-dose gluten on NCGS in order to avoid the non-specific effects of gastrointestinal fermentation and to avoid causing death in normal individuals, which could be induced by high-dose gluten. Subjective symptom score and haematological index were used as criteria. The symptoms of NCGS included depression and aphthous ulcers. ${ }^{4}$ Peters et al. reported that GFD in patients with non-coeliac gluten sensitivity led to amelioration of depression. ${ }^{5}$

Patients with RAS may also suffer from anaemia or haematinic (vitamin B12 and folic acid) deficiency, a diet containing vitamin B12 and folic acid or one beneficial for anaemia could reduce the recurrence of RAS. Kozlak et al. surveyed 100 subjects through a diet history questionnaire (excluding people with systemic diseases closely associated with RAS); the information included the frequency and total intake of 124 types of foods in the past 365 days. The NHANES (National Health and Nutrition Examination Survey) database was used as control and Dietcalc software programme was used to calculate the nutrient density. The results suggested that RAS may be the only clinical manifestation of early vitamin B12 and folic acid deficiency. ${ }^{6}$ Our meta-analysis also revealed that anaemia or potential anaemia (deficiency of vitamin B12, folic acid, etc.) is an important factor in RAS. ${ }^{7}$ Of course, the diet is not limited to the food rich in vitamin B12 or folic acid, there are other micronutrients that are also conducive to haematopoiesis. Of note, a number of studies have reported that both folate and vitamin B12 contribute to mental health as well.

Insufficient and irregular sleep patterns might induce and/or aggravate RAS in college students, which could possibly be associated with disturbances in the diurnal secretion cycles of hormones like growth hormone and cortisol. ${ }^{8}$ Using multiple time point data from a prospective study, Tochigi et al. observed both a correlation and an interaction between delayed bedtime and depression/anxiety. ${ }^{9} \mathrm{~A}$ large number of studies have suggested a relationship between depression and delayed sleep. Sakamoto et al. reported that sleep duration mediated the effect of delayed bedtime on the symptoms of depression; if people feel they have had sufficient sleep, delayed bedtime does not appear to be related to depression. However, patients with depression might not think that they have had adequate sleep, so the association between the two is not completely clear. ${ }^{10}$

In summation, some lifestyle habits seem to be associated with RAS or RAS-type ulceration as well as mental health. Although it is the commonest oral mucosal disease, the aetiology of RAS remains undefined. Immune mechanisms are currently considered a major influencing factor, but the psychological state of RAS patients, which is different from the general population, should also not be ignored. Patients with RAS present with a higher degree of stress and anxiety, this can be evaluated by measuring cortisol concentrations in saliva and blood. ${ }^{11}$ Huling et al. followed-up patients with RAS for one year via telephone. An automated telephone system was used to call and assess patients every week. If the answer was a simple 'yes' or 'no', it was recorded by instructing patients to press a corresponding button. Answers that required a verbal description were recorded and analysed by two researchers. The study found a close correlation between psychological stress and the onset and duration of RAS. ${ }^{12,13}$ Stress appears to be one of the major predisposing factors to all immune-mediated conditions, including RAS. In common parlance in China, RAS is equated with the 'get-the-fire-evil' (a term from CTM). In CTM, the heart matches fire. The Inner Canon of the Yellow Emperor states, 'All kinds of diseases with pain, itching and sores are exclusively related to the heart'.

Mental health might play a core role in RAS and all habits contributing to mental health might help in reduction of symptoms. We could draw lessons from cognitive studies; for example, Forman et al. conducted randomised controlled trials and reported that lifestyle changes could delay the onset and ease the symptoms of stress in female subjects. These changes included dietary approaches to stop hypertension (DASH) diet, intake of supplemental folic acid, and vigorous exercises. ${ }^{14}$ The research of Cayea et al. revealed that resistance exercises, even at a low frequency of 1-2 times a week, slowed the loss of cognitive function among the elderly, and recommended that they should be part of their regimen. ${ }^{15}$

Despite the lack of prospective studies on a large patient population with a long follow-up to provide the best evidence, it can still be argued that lifestyle changes are the first and most important step for reduction and control of RAS. In this manner, drug use and their incident adverse reactions can be minimised, which can be especially important to children, patients already on poly-therapy (for example, the elderly), pregnant women, and other special populations. Prevention of recurrence could also reduce the number of hospital visits and save physician work-hours.

In conclusion, I am of the opinion that lifestyle changes could benefit patients with RAS and that practitioners of oral medicine should recommend a healthy lifestyle to patients before prescribing medicines.

1. Brocklehurst $P$, Tickle M, Glenny A M et al. Systemic interventions for recurrent aphthous stomatitis (mouth ulcers). Cochrane Database Syst Rev 2012; 9: CD005411.

2. Sotos-Prieto M, Bhupathiraju S N, Mattei J et al. Association of Changes in Diet Quality with Total and CauseSpecific Mortality. N Engl J Med 2017; 377: 143-153.

3. Ferguson R, Basu M K, Asquith P, Cooke W T. Jejunal mucosal abnormalities in patients with recurrent aphthous ulceration. Br Med J 1976; 1: 11-13.

4. Di Sabatino A, Volta U, Salvatore C et al. Small Amounts of Gluten in Subjects With Suspected Nonceliac Gluten Sensitivity: A Randomized, Double-Blind, PlaceboControlled, Cross-Over Trial. Clin Gastroenterol Hepatol 2015; 13: 1604-1612.

5. Peters S L, Biesiekierski J R, Yelland G W, Muir J G, Gibson PR. Randomised clinical trial: gluten may cause depression in subjects with non-coeliac gluten sensitivit - an exploratory clinical study. Aliment Pharmacol Ther 2014; 39: 1104-1112.

6. Kozlak S T, Walsh S J, Lalla R V. Reduced dietary intake of vitamin $\mathrm{B} 12$ and folate in patients with recurrent aphthous stomatitis. J Oral Pathol Med 2010; 39: 420-423.

7. Chen $\mathrm{H}$, Sui $\mathrm{Q}$, Chen Y, Ge L, Lin M. Impact of haematologic deficiencies on recurrent aphthous ulceration: a meta-analysis. Br Dent J 2015; 218: E8.

8. Ma R, Chen $\mathrm{H}$, Zhou T et al. Effect of bedtime on recurrent aphthous stomatitis in college students. Oral Surg Oral Med Oral Pathol Oral Radiol 2015; 119: 196-201.

9. Tochigi M, Usami S, Matamura M et al. Annual longitudinal survey at up to five time points reveals reciprocal effects of bedtime delay and depression/anxiety in adolescents. Sleep Med 2016; 17: 81-86.

10. Sakamoto N, Nanri A, Kochi T et al. Bedtime and sleep duration in relation to depressive symptoms among Japanese workers. J Occup Health 2013; 55: 479-486.

11. Soto Araya M, Rojas Alcayaga G, Esguep A. Association between psychological disorders and the presence of Oral lichen planus, Burning mouth syndrome and Recurrent aphthous stomatitis. Med Oral 2004; 9: 1-7.

12. Albanidou-Farmaki E, Poulopoulos A K, Epivatianos A, Farmakis K, Karamouzis M, Antoniades D. Increased anxiety level and high salivary and serum cortisol concentrations in patients with recurrent aphthous stomatitis. Tohoku J Exp Med 2008; 214: 291-296.

13. Huling L B, Baccaglini L, Choquette L, Feinn R S, Lalla $R V$. Effect of stressful life events on the onset and duration of recurrent aphthous stomatitis. J Oral Pathol Med 2012; 41: 149-152.

14. Cayea $D$, Eckstrom $E$, Christmas $C$. Update in geriatric medicine. J Gen Intern Med 2012; 27: 371-375.

15. Forman J P, Stampfer M J, Curhan G C. Diet and lifestyle risk factors associated with incident hypertension in women. JAMA 2009; 302: 401-411. 